\title{
Stationary phase type and temperature effect on HPLC separation of lactic acid enantiomers
}

\author{
Laura Jánovová, Katarína Hroboňová \\ Slovak University of Technology in Bratislava, Faculty of Chemical and Food Technology, \\ Institute of Analytical Chemistry, \\ Radlinského 9, SK-812 37 Bratislava, Slovak Republic \\ katarina.hrobonova@stuba.sk
}

\begin{abstract}
Lactic acid is a biologically important organic acid existing in two enantiomeric forms which are differently metabolized in the human body. In this paper, direct chiral separation of lactic acid by high performance liquid chromatography is presented. Five chiral stationary phases based on macrocyclic antibiotics were used for enantioseparation and chromatographic parameters, such as retention factors, resolution and selectivity factors, were determined under different column temperatures ranging from 5 to $45^{\circ} \mathrm{C}$. Optical isomers of lactic acid were efficiently separated using chiral stationary phases based on teicoplanin $\left(R_{\mathrm{S}}=1.9\right)$ and ristocetin $\left(R_{\mathrm{S}}=1.7\right)$ in reversed-phase separation mode at the column temperature of $25^{\circ} \mathrm{C}$.
\end{abstract}

Keywords: chiral separation, high performance liquid chromatography, lactic acid, macrocyclic antibiotics, temperature

\section{Introduction}

Lactic acid (2-hydroxypropanoic acid) is a biologically important organic acid that was first discovered in sour milk. It is an $\alpha$-hydroxy acid with a chiral carbon atom and thus it exists in two enantiomers $\mathrm{L}(+)$ and $\mathrm{D}(-)$ (Fig. 1) (Norton et al., 2006; Martinez et al., 2013). In the biological metabolic processes, $\mathrm{L}(+)$-lactic acid is present as a metabolic intermediate, while the $\mathrm{D}(-)$ isomer is excreted from the body. $\mathrm{D}(-)$-lactic acid is produced by plants, bacteria, and some types of algae (Norton et al., 2006). Both enantiomeric forms have been reported for different applications in food processing, chemical, and cosmetic industry. Due to their different effects, e.g. $\mathrm{D}(-)$ enantiomer is used for chemical production of some plasticizers, adhesives and as a component of many household cleaners, while $\mathrm{L}(+)$ enantiomer has many applications in the field of skin care. Approximately $70 \%$ of $\mathrm{L}(+)$-lactic acid produced is used in the food processing industry as a preservative (in cheese, beverages, and others), antioxidant, $\mathrm{pH}$ regulator and flavoring agent (Vargas et al., 2016). Owing to its moisturizing, antimicrobial and rejuvenating effects on the skin, lactic acid is used in hygiene and esthetic products as well as in oral hygiene products. Lactic acid reduces irregular skin pigmentation and helps with clogged sebaceous pores (Abd Alsahebet et al., 2015; Rodrigues et al., 2017). Its concentration in cosmetics products usually ranges from $1 \%$ to $10 \%$ and the $\mathrm{pH}$ value of these cosmetic products should not be lower than 3.5. Maximum concentration for small consumers should not exceed $20 \%$ (Huang et al., 2002; Abd
Alsahebet et al., 2015). Changes in the $\mathrm{pH}$ of the product or an inappropriate enantiomeric form may cause negative effects such as rashes, irritation, burning, redness, and changes in sensitivity to sunlight (Huang et al., 2001). Therefore, it is necessary to assure the safety of consumers by monitoring parameters such as $\mathrm{pH}$ value, concentration, and optical form of lactic acid (Dutra et al., 2006).<smiles>C[C@@H](O)C(=O)O</smiles>

$\mathrm{L}(+)$ enantiomer<smiles>C[C@@H](O)C(=O)O</smiles>

D(-) enantiomer
Fig. 1. Chemical structures of $\mathrm{L}(+)$ and $\mathrm{D}(-)$-lactic acid.

Several achiral analytical methods for the determination of organic acids, including $\alpha$-hydroxy acids, have been developed: volumetric methods (alkalimetry) (Feng et al., 2020), colorimetric (Nicoletti et al., 1999), spectrophotometric (Borshchevskaya et al., 2016), electromigration (Dutra et al., 2006) and chromatographic methods (gass chromatography) (Hušek et al., 2003) or high performance liquid chromatography (Yates et al., 1999). Determination of enantiomeric forms can be realized by liquid chromatography (Norton et al., 2007; Henry et al., 2012), gas chromatography (Ding et al., 2018) or by capillary electrochromatography methods (Mohr et al., 2012). The choice of a suitable method 
depends on the nature of the acids analyzed, their relative concentration, as well as on the nature of the sample matrix.

High performance liquid chromatography with UV detection (wavelength of $210 \mathrm{~nm}$ ) (Okubo et al., 2000) as well as mass spectrometry detection (Henry et al., 2012), preferably in direct approach using chiral stationary phases (CSP), is the most widely used method for lactic acid enantiomers separation. The choice of direct HPLC is preferred for enantioselective separation regarding its high efficiency. Direct chromatographic enantioseparation is based on the formation of transient diastereomeric complexes between functional groups belonging to the analyte and chiral selectors of the stationary phase. Therefore, one of the two complexes formed is more stabilized by means of potential intermolecular interactions (e.g. hydrogen bonding, $\pi-\pi$ complexation, dipole stacking, ionic and steric interactions, inclusion onto cavities) and retained on CSP. HPLC columns in ligand exchange mode (Henry et al., 2012; Okubo et al., 2000) and macrocyclic antibiotic in polar organic mode (Norton et al., 2007) have been used for enantioseparation of lactic acid enantiomers.

The use of macrocyclic glycopeptides-based chiral stationary phases in reversed phase separation mode is reported here for lactic acid enantiomers separation. Studying the effect of chiral selector type and column temperature on enantioresolution will contribute to understanding chiral recognition strategies.

\section{Materials and Methods}

\section{Chemicals and solvents}

Standard of DL-lactic acid (90\%) was purchased from Sigma-Aldrich (St. Louis, USA) and L-lactic acid $(98 \%)$ was obtained from Alfa Aesar (Germany). Chemicals used for mobile phase preparation were acetonitrile (HPLC gradient grade) obtained from Centralchem (Slovakia) and ammonium acetate from Lachema (Czech Republic). Deionized water was obtained using an AquaMax ultra (series 370) water purification system.

\section{HPLC}

HPLC separation was carried out on an Agilent Technologies high performance liquid chromatographic system (series 1100) equipped with a binary solvent delivery pump, injector valve, column thermostat, and a diode array detector (DAD). Chromatographic separation was conducted using analytical columns with macrocyclic antibiotic stationary phases based on chiral selectors ristocetin (Chirobiotic R), vancomycin (Chirobiotic V), teicoplanin (Chirobiotic T), teicoplanin aglycone (Chirobiotic TAG), and methyl- ated teicoplanin aglycone (Chirobiotic Methyl-TAG). Column parameters were as follows: I.D. of $250 \times 4.6 \mathrm{~mm}$ (length $\times$ interanal diameter) and particle size of $5 \mu \mathrm{m}$ for Chirobiotic R, Chirobiotic V, Chirobiotic T, Chirobiotic TAG columns, and I.D. of $150 \times 4.6 \mathrm{~mm}$ (length $\times$ interanal diameter) and particle size of $5 \mu \mathrm{m}$ for the Chirobiotic Methyl-TAG column. Isocratic elution with binary mobile phase consisting of acetonitrile and $0.03 \mathrm{~mol} / \mathrm{L}$ solution of ammonium acetate $(\mathrm{pH}=7)(85: 15, \mathrm{v} / \mathrm{v})$ was used for separation. Column temperature ranging from $5{ }^{\circ} \mathrm{C}$ to $45^{\circ} \mathrm{C}$ (increment of $5{ }^{\circ} \mathrm{C}$ ) was tested for temperature study. Injection volume was $20 \mu \mathrm{L}$ and the flow rate of the mobile phase was $1.0 \mathrm{~mL} / \mathrm{min}$. DAD was operated in the wavelength range of 190-400 nm, and detection wavelength was set to $210 \mathrm{~nm}$. Initial screening of enantioselective stationary phases was performed for DL- and L-lactic acid solutions prepared by dissolving standards in the mobile phase (concentration of $1 \mathrm{mg} / \mathrm{mL}$ ).

\section{Results and Discussion}

Selection of CSP, separation mode and column temperature are crucial for efficient enantioseparation in HPLC. The aim of this study was to select suitable chromatographic conditions for direct HPLC separation of lactic acid enantiomers. The initial stage was focused on identifying the chiral selector type and column parameters in order to determine their influence on the chromatographic characteristics (retention factors, resolution, selectivity factors and high equivalent of theoretical plates). The chiral selectors of macrocyclic antibiotic type, their aglycones and derivatives in reversed-phase mode were chosen for the enantiomers separations. Several compositions of the mobile phase were tested by changing the nature and percentage of the organic modifier (methanol or acetonitrile), water or ionic modifier solution (acetic acid, triethylamine and ammonium acetate, $\mathrm{pH}$ was varied) to achieve efficient and fast enantioseparation. As a result of the screening experiments, suitable mobile phase composition was set as acetonitrile $-0.03 \mathrm{~mol} / \mathrm{L}$ of ammonium acetate $(\mathrm{pH}=7)(85: 15, \mathrm{v} / \mathrm{v})$ for all tested CSP.

\section{Effect of stationary phase type on enantioresolution Among existing CSPs, macrocyclic antibiotic stationary phases have been extensively used in reversed-phase, normal-phase, and polar-organic mode HPLC for efficient resolution of chiral com- pounds (Ilisz et al., 2012; Armstrong et al., 1994). Selection of CSP and structure of the chiral selector play an important role in HPLC separation of enan- tiomers. Five types of CSPs containing chiral selec- tors ristocetin, vancomycin, teicoplanin, teicoplanin}




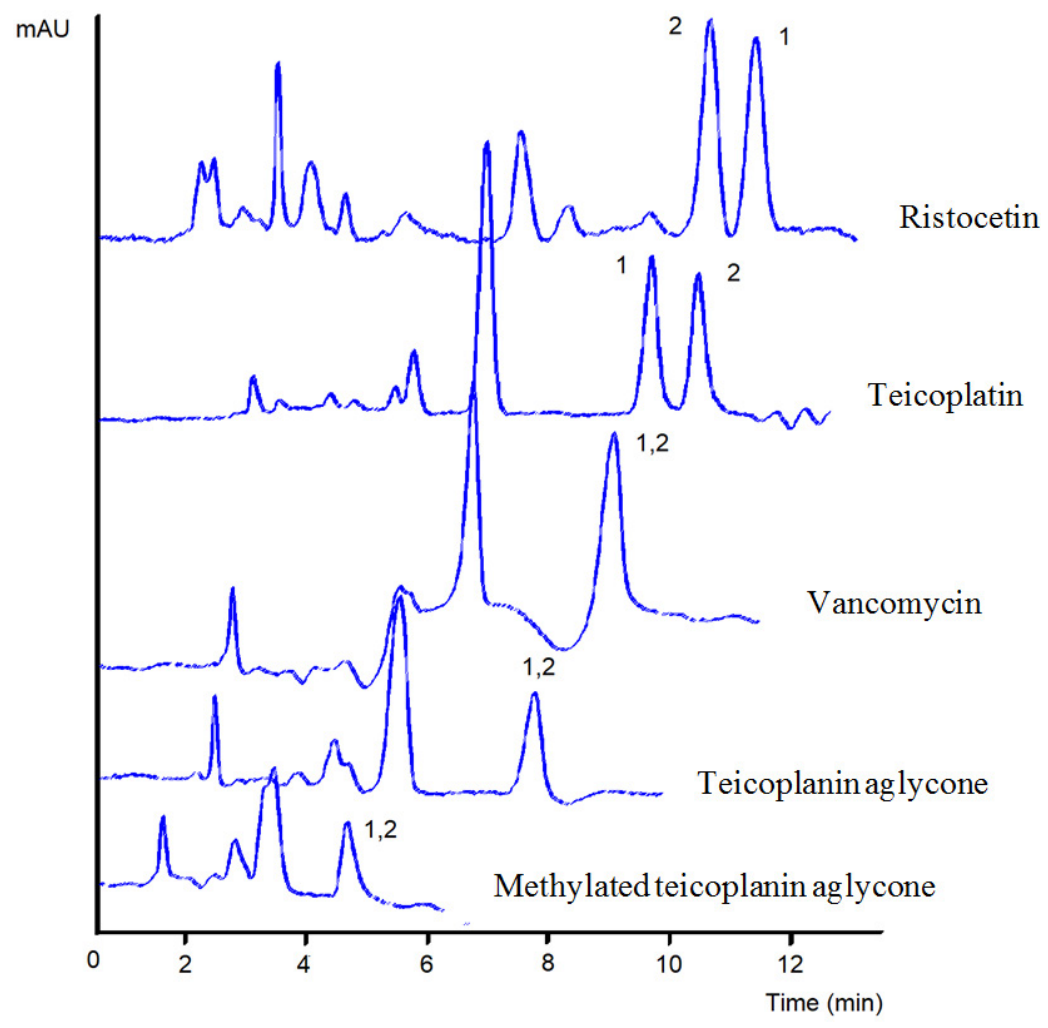

Fig. 2. HPLC chromatograms of lactic acid enantiomers using macrocyclic glycopeptide chiral selectors. Chromatographic conditions: mobile phase: acetonitrile/0.03 mol/L of ammonium acetate (85:15, v/v), temperature: $25^{\circ} \mathrm{C}$, flow rate: $1 \mathrm{~mL} / \mathrm{min}$, detection: $\mathrm{DAD}(\lambda=210 \mathrm{~nm})$;

Legend: 1 - L-lactic acid, 2 - D-lactic acid.

aglycone and methylated teicoplanin aglycone were screened to achieve resolution of lactic acid enantiomers. Fig. 2 shows chromatograms of DL-lactic acid using tested CSPs (other peaks are unknown impurities present in the analyzed standard solution). Baseline separation of enantiomers (resolution values above 1.6) and good enantioselectivity were obtained in ristocetin CSPs at analysis times up to 15 min. Teicoplanin CSP displayed good enantioselectivity and lower retention time. Vancomycin and teicoplanin aglycone CSPs did not resolve the enantiomers under operating reversed-phase conditions selected for screening. Table 1 presents the calculated chromatographic characteristics, retention factor, selectivity coefficient, resolution, and high equivalent of theoretical plate. While the ristocetin based CSP showed the highest retention of enantiomers (the highest values of $k_{\mathrm{L}}, k_{\mathrm{D}}$ ) and higher column efficiency (the lowest value of high equivalent of theoretical plate), the teicoplanin CSP exhibited higher enantioselectivity as well as higher enantiorecognition ability (higher value of resolution).

These differences in the chiral recognition mechanism of macrocyclic antibiotic chiral selectors result from their different structures and func- tionalities (Table 2) (Ilisz et al., 2012; Armstrong et al., 1994). Since the chiral stationary phase contains several ionizable groups, such as amines, amides, carboxylic acids, and phenols, sugar moieties and cavities, retention of enantiomers based on electrostatic, hydrogen bonds, $\pi-\pi$, steric, or inclusion interactions is possible. The nature and functionalities of a chiral selector influences the formation of diastereomeric complexes, analyte-CSP, which consequently affects their enantioresolving ability. Enantioselectivity of teicoplanin and ristocetin SCPs to resolve lactic acid enantiomers results from the attached sugar units. It is evident (Table 2) that molecules of teicoplanin and ristocetin contain several sugar units, which positively affects the enantiorecognition, in contrast to vancomycin in which the aglycone bears a disaccharide unit. The results obtained for teicoplanin based chiral stationary phases (Chirobiotic T, Chirobiotic TAG, Methyl-TAG) indicate that sugar units play a dominant role in the enantiocognition. Separation of enantiomers was achieved in teicoplanin CSP while teicoplanin aglycone CSP and its modified methylated version did not show the ability to separate enantiomers of lactic acid in reversed- 
Tab. 1. Chromatographic characteristics for HPLC separation of lactic acid enantiomers using macrocyclic glycopeptide CSPs.

\begin{tabular}{lccccc}
\hline & $\begin{array}{c}\boldsymbol{t}_{\mathrm{RL}} / \boldsymbol{t}_{\mathrm{RD}} \\
(\mathbf{m i n})\end{array}$ & $\boldsymbol{k}_{\mathrm{L}} / \boldsymbol{k}_{\mathrm{D}}$ & $\boldsymbol{\alpha}$ & $\boldsymbol{R}_{\mathrm{S}}$ & $\begin{array}{c}\boldsymbol{H}_{\mathrm{L}} / \boldsymbol{H}_{\mathrm{D}} \\
(\boldsymbol{\mu m})\end{array}$ \\
\hline Ristocetin & $11.42 / 10.66$ & $2.64 / 2.40$ & 1.10 & 1.7 & $28.1 / 26.8$ \\
Teicoplanin & $9.52 / 10.31$ & $1.78 / 2.02$ & 1.13 & 1.9 & $30.6 / 28.0$ \\
Vancomycin & 9.13 & 1.68 & 1.00 & 0 & 67.8 \\
Teicoplanin aglycone & 7.81 & 1.66 & 1.00 & 0 & 59.3 \\
Methylated teicoplanin aglycone & 4.65 & 1.42 & 1.00 & 0 & 84.0 \\
\hline
\end{tabular}

${ }^{1} t_{\mathrm{R}}-$ retention time of enantiomer, $k_{\mathrm{D}}-$ retention factor of enantiomer, $R_{\mathrm{S}}-$ resolution, $\alpha-$ selectivity coefficient, $H-$ high equivalent of theoretical plate, RSD\% $\leq 2.13 \%(n=3)$, HPLC conditions: see Fig. 2.

Tab. 2. Structural properties of macrocyclic antibiotic chiral selectors used in this study.

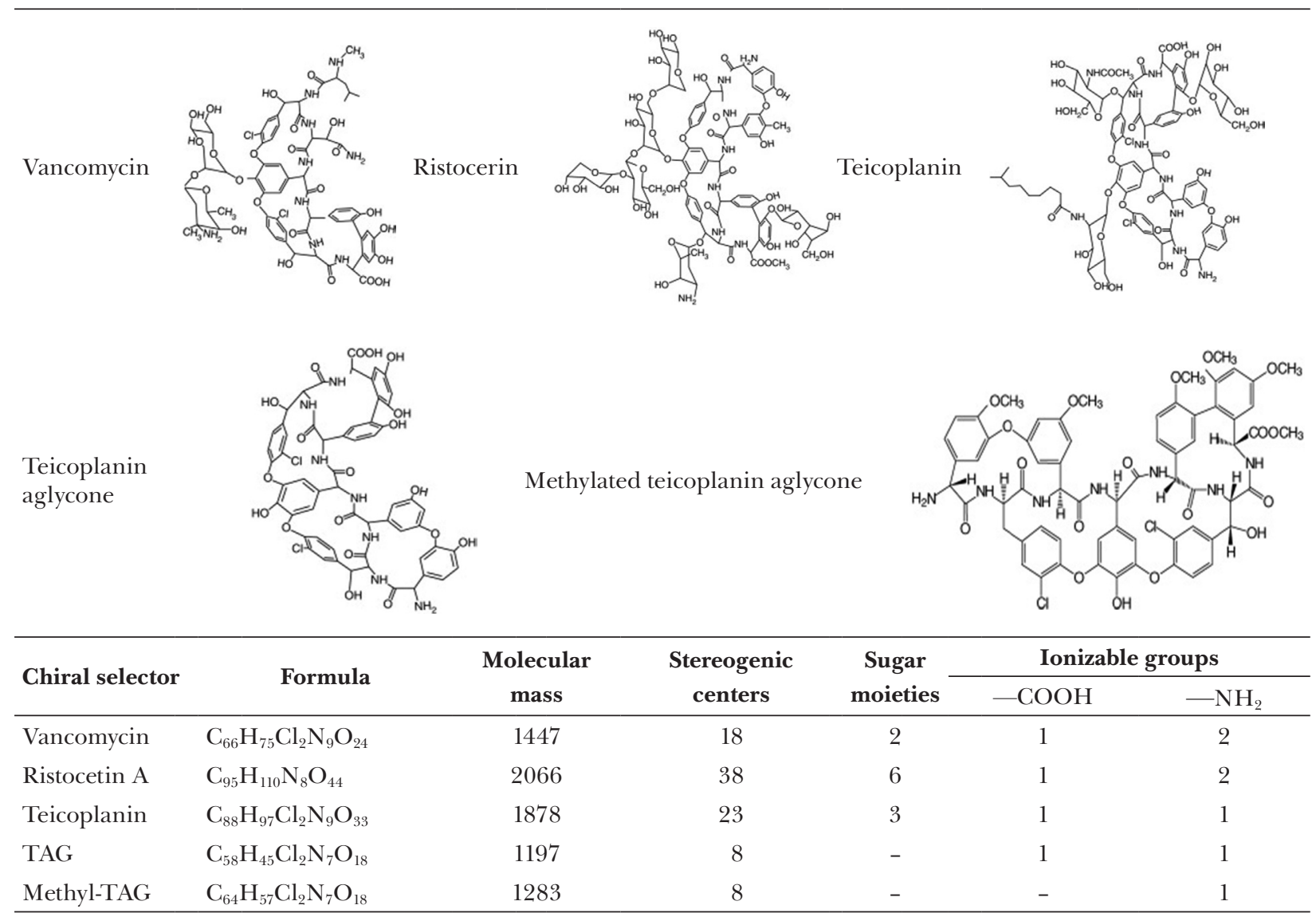

(D’Acquarica et al., 2008; Ilisz et al., 2012)

phase mode with the mobile phase acetonitrile/ ammonium acetate. Based on the structure information of the CSPs used, it is evident that selectors with a larger number of stereogenic centers (38 for ristocetin), aromatic rings ( 7 for ristocetin), hydroxyl groups (21 for ristocetin) and more sugar units (more than 2) are likely to be more effective in the separation of carboxylic acid enantiomers.

These results are complementary to the previous work of Norton et al. focused on lactic acid enantiomers separation in a TAG chiral column in polar organic mode (Norton et al., 2007).
Mobile phase used for enantioseparation consists of acetonitrile and ammonium acetate, indicating possible intermolecular interactions between the analyte and chiral selector. Considering functional groups of CSPs, the interactions of the analyte and the polar groups in CSPs are involved in binding mechanisms. Transient diastereomers are formed through hydrogen bonds using the $\mathrm{O}-\mathrm{H}, \mathrm{C}=\mathrm{O}$ and $\mathrm{N}-\mathrm{H}$ groups and through dipole-dipole interactions using the $\mathrm{C}=\mathrm{O}$ groups. Hydrogen bonds between the analyte and the chiral selector can be relatively weak in the reversed-phase separation mode mainly due to the competitive effect 


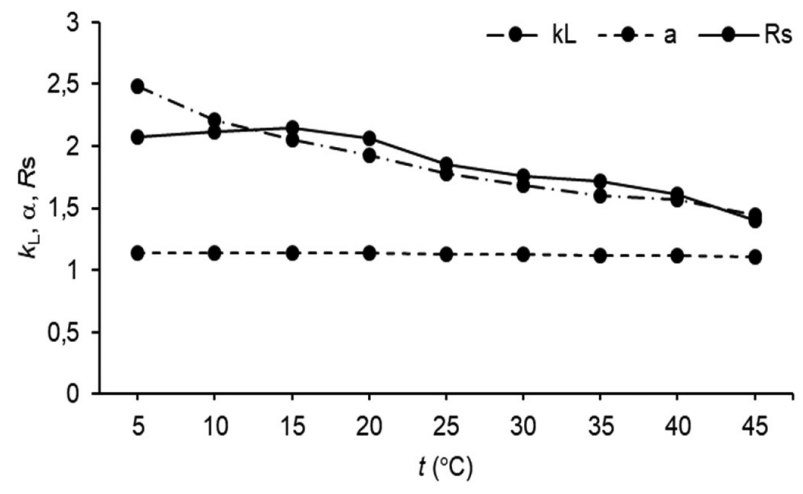

a)

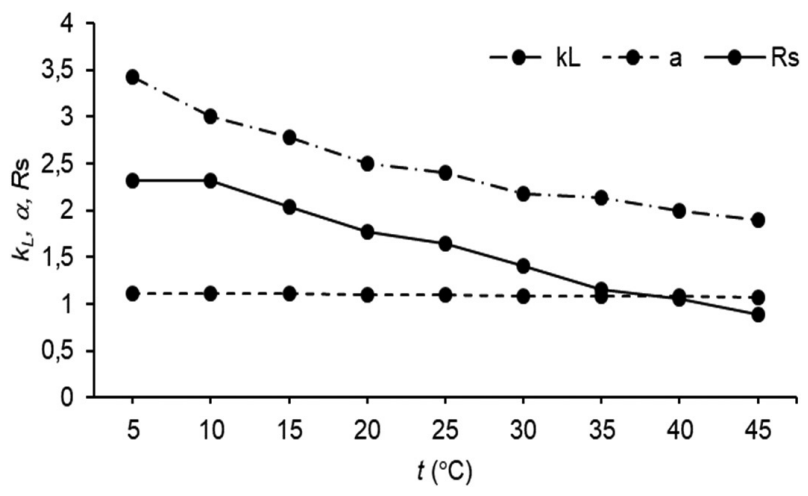

b)

Fig. 3. Effect of column temperature on chromatographic characteristics of lactic acid enantioseparation in teicoplanin (a) and ristocetin (b) based CSP.

of methanol as a mobile phase component (Xiao et al., 2006; Ilisz et al., 2012; Armstrong et al., 1994), therefore, acetonitrile as an organic modifier was used in this work. Lactic acid provides $\mathrm{C}-\mathrm{OH}$ and $\mathrm{C}=\mathrm{O}$ functional groups which form hydrogen bonds with the carbamate groups in CSPs. In addition, electrostatic interactions (lactic acid $\mathrm{pKa}=3.9)$ with stationary phase amines may also be involved in chiral recognition. These interactions are promoted by $\mathrm{pH}$ of the mobile phase facilitating lactic acid ionization to the respective conjugate base. These results suggest that lactic acid was enantioseparated by a combination of interactions involving both the aglycone basket and its attached sugar units.

\section{Effect of temperature on enantioresolution}

Temperature affects the separation of enantiomers in chiral HPLC significantly. In this work, the effect of column temperature on the retention and resolution of lactic acids enantiomers was investigated in the temperature range of $5-45^{\circ} \mathrm{C}$.

As it is shown in Figure 3, increasing the column temperature caused a decrease in the retention factor values of enantiomers in both chromatographic columns tested (similar trend was obtained for other CSPs under study, results not shown). Mutual retention of analytes expressed by selectivity coefficients varied moderately with temperature (1.11-1.14 for teicoplanin CSP, 1.07-1.11 for ristocetin CSP). Decrease in selectivity coefficients and resolution values was observed at higher temperatures (Fig. 3). It was also found that changes in the column temperature within the test interval do not influence the elution order of enantiomers. Efficient resolution of enantiomers (resolution value higher than 1.5) was achieved at temperatures lower than $25^{\circ} \mathrm{C}$ and $40{ }^{\circ} \mathrm{C}$ for ristocetin and teicoplaninbased columns, respectively.

\section{Conclusions}

In this study, various types of macrocyclic glycopeptide-based chiral stationary phases were tested for chiral HPLC separation of lactic acid in reversed-phase mode. Separation of enantiomers has been observed in stationary phases with ristocetin and teicoplanin chiral selectors. These results suggest that a combination of interactions involving both the aglycone basket and its attached sugar units are responsible for enantiorecognition. Investigating the effect of chiral selector type on enantioresolution will contribute to understanding chiral recognition strategies. The type of column together with column temperature significantly influenced the resolution of enantiomers (statistically significant, $\alpha=0.05$ ). Efficient separation of enantiomers was obtained at laboratory and lower column temperatures.

\section{Acknowledgement}

This research was financially supported by the Scientific Grant Agency of the Ministry of Education of the Slovak Republic and the Slovak Academy of Sciences (grant No. VEGA 1/0159/20). The authors are grateful to professor D. W. Armstrong for providing chiral stationary phases.

\section{References}

Abd Alsaheb RA, Aladdin A, Othman NZ, Abd Malek R, Leng OM, Aziz R, Enshasy HA (2015) J. Chem. Pharm. Res. 7(10): 729-735.

Armstrong DW, Tang Y, Chen S, Zhou Y, Bagwill C, Chen JR (1994) Anal. Chem. 66: 1473-1484.

Borshchevskaya LN, Gordeeva TL, Kalinina AN, Sineokii SP (2016) J. Anal. Chem. 71(8): 755-758.

D’Acquarica I, Gasparrini F, Misiti D, Pierini M, Villani C (2008) Adv. Chromatogr. (N.Y.) 46: 109-173.

Ding X, Lin S, Weng H, Liang J (2018) J. Sep. Sci. 41(12): 2576-2584. 
Dutra EA, Santoro MIRM, Micke GA, Tavares MFM, Kedor-Hackmann ERM (2006) J. Pharm. Biomed. Anal. 40(2): 242-248.

Feng S, Xiang S, Bian X, Li G (2020) Microch. J. 157: 105049.

Henry H, Marmy Conus N, Steenhout P, Béguin A, Boulat O (2012) Biomed. Chromatogr. 26(4): 425-428.

Huang WS, Lin CC, Huang MC, Wen KC (2002) J. Food Drug Anal. 10(2): 95-100.

Hušek P, Šimek P, Matucha P (2003) Chromatographia 58: 623-630.

Ilisz I, Pataj Z, Aranyi A, Péter A (2012) Sep. Purif. Rev. 41(3): 207-249.

Martinez FAC, Balciunas EM, Salgado JM, Gonzalez JMD, Converti A, de Souza Oliveira RP (2013) Trends Food Sci. Technol. 30(1): 70-83.

Mohr S, Sepic G, Schmid MG (2012) Croat. Chem. Acta 85(1): 33-36.
Nicoletti I, Corradini C, Cogliandro E, Cavazza A (1999) Int. J Cosmet. Sci. 21(4): 265-274.

Norton D, Crow B, Bishop M, Kovalcik K, George J, Bralley JA (2007) J. Chromatogr. B 850(1-2): 190-198.

Okubo S, Mashige F, Omori M, Hashimoto Y, Nakahara K, Kanayawa H, Matsushima Y (2000) Biomed. Chromatogr. 14(7): 474-477.

Rodrigues C, Vandenberghe LPS, Woiciechowski AL, de Oliveira J, Letti LAJ, Soccol CR (2017) Current Developments in Biotechnology and Bioengineering: Production, Isolation and Purification od Industrial Products, Elsevier, pp. 543-556.

Vargas E, Ruiz MA, Campuzano S, de Rivera GG, López-Colino F, Reviejo AJ, Pingarrón JM (2016) Talanta 152: 147-154.

Xiao TL, Tesarova E, Anderson JL, Egger M, Armstrong DW (2006) J. Sep. Sci. 29(3): 429-445.

Yates RL, Havery DC (1999) J. Cosmet. Sci. 50: 315-325. 